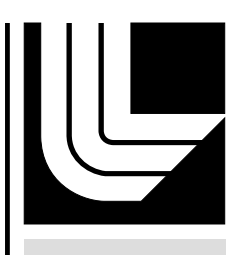

LAWRENCE LIVERMORE N A TIO N A L LABORATORY

\title{
Advances in Optical Spectroscopy and Imaging of Breast Lesions
}

S. Demos, A. J. Vogel, A. H. Gandjbakhche

January 5, 2006

Journal of Mammary gland biology and neoplasia 
This document was prepared as an account of work sponsored by an agency of the United States Government. Neither the United States Government nor the University of California nor any of their employees, makes any warranty, express or implied, or assumes any legal liability or responsibility for the accuracy, completeness, or usefulness of any information, apparatus, product, or process disclosed, or represents that its use would not infringe privately owned rights. Reference herein to any specific commercial product, process, or service by trade name, trademark, manufacturer, or otherwise, does not necessarily constitute or imply its endorsement, recommendation, or favoring by the United States Government or the University of California. The views and opinions of authors expressed herein do not necessarily state or reflect those of the United States Government or the University of California, and shall not be used for advertising or product endorsement purposes. 


\title{
Advances in Optical Spectroscopy and Imaging of
}

\section{Breast Lesions}

\author{
Stavros G. Demos ${ }^{1}$, Abby J. Vogel ${ }^{2}$, Amir H. Gandjbakhche ${ }^{2}$ \\ 1 Lawrence Livermore National Laboratory, 7000 East Ave., Livermore, CA 94551 and, \\ UC Davis Medical School, Medical Center, 4860 Y St., Suite 2200, Sacramento, CA 95817 \\ 2 Laboratory of Integrative and Medical Biophysics, National Institutes of Health, 9000 \\ Rockville Pike, Building 9, Room B1E05, Bethesda, Maryland 20892
}

\begin{abstract}
:
A review is presented of recent advances in optical imaging and spectroscopy and the use of light for addressing breast cancer issues. Spectroscopic techniques offer the means to characterize tissue components and obtain functional information in real time. Three-dimentional optical imaging of the breast using various illumination and signal collection schemes in combination with image reconstruction algorithms may provide a new tool for cancer detection and monitoring of treatment.
\end{abstract}




\section{Chapter 1. Background}

According to the National Institutes of Health, breast cancer is the most diagnosed nonskin cancer and the second leading cause of cancer death among women in the United States. Although the breast cancer diagnosis rate has increased, there has been a steady drop in the overall breast cancer death rate since the early 1990s. Depending on the size of the tumor and the involvement of the lymph nodes, survival rates can vary from $45-95 \%$. The key problem with the breast is the variation in demographic size, texture, chemical composition, and age. X-ray mammography is the gold standard for breast cancer screening and detection. Mammography is most sensitive in women over 35-40 years of age because of their fatty breast composition ${ }^{1}$ and less effective and sensitive in younger women because they have denser breasts ${ }^{2}$. For younger women, ultrasound is commonly used. Magnetic resonance imaging (MRI) can also play a significant role in the diagnosis and characterization of breast disease.

According to the National Cancer Society, up to $10 \%$ of all breast cancers, roughly 20,000 cases per year in the U.S., are not discovered by X-ray mammography. Also, X-ray mammography uses ionizing radiation and requires uncomfortable breast compression. It also suffers from a significant number of false positives that often lead to unnecessary biopsy since biopsy is generally required to determine malignancy in most women with an abnormal mammogram. All three techniques, X-ray mammography, ultrasound, and MRI provide high spatial resolution, but comparatively little information about molecular-level changes in breast tissue $^{1,3}$. Consequently, new detection technologies are needed that can overcome current limitations.

Photonic technology plays an important role in the development of new medical diagnostic and therapeutic instruments. Readily available compact solid-state lasers and various 
types of light sources allow one to examine tissues in a clinical environment using new and emerging photonic technologies. Major advances in light array detectors provide high signal sensitivities that enable new imaging approaches previously technologically infeasible. In addition, since the computation power of personal computers has been continuously increasing, signal processing speed and image reconstruction algorithms are also advancing. Key benefits of using light for medical imaging are that light is non-ionizing radiation, potentially much cheaper, and can be delivered to a localized region by using either an external source or internally through small optical fibers.

Optical imaging and optical biopsy are two of the research areas where rapid progress has been achieved over the past ten years. The aim of optical imaging is to provide three-dimensional mapping of tissue structures using light. Photon selection and image reconstruction techniques are implemented to improve image contrast and resolution. Optical biopsy uses the difference in the optical properties of different tissue components to obtain information regarding the tissue makeup, status, and composition. Optical biopsy methods to detect breast cancer in vivo have been explored by various research groups. Such optical biopsy methods have demonstrated high sensitivity for cancer detection in various parts of the body. Cancer specific optical "signatures" must be present in order to devise optical biopsy methods for cancer detection. These optical "signatures" may arise from differences in the biochemical and/or structural characteristics of the tissues.

The development of an optical mammography system able to image the breast and detect tumors has been a focus area in the field of optical imaging. Although significant progress has been achieved in this very challenging area, preliminary results have documented the potential of optical imaging for noninvasive breast cancer detection. In recent years, near infrared (NIR) 
spectroscopy and tomography have been explored by numerous research groups as a means of detecting and characterizing breast cancer. Many groups have demonstrated an ability to accurately quantify hemoglobin absorption and other intrinsic tissue chromophore concentrations (i.e. fat or water) and scattering properties, thereby providing valuable functional information. Spatial and temporal contrasts in these properties may be uniquely useful for diagnosing disease.

The objective of this work is to provide a comprehensive review of the progress of photonics methods to address breast cancer issues. This review is divided into three major focus areas: a) optical spectroscopy methods suitable for tissue evaluation in real time; b) noninvasive NIR spectroscopy for the acquisition of functional information; and c) optical imaging methods for functional tumor characterization.

\section{Chapter 2. Optical biopsy methods}

The term "optical biopsy" describes the use of optical spectroscopy to characterize tissue, and requires direct exposure of the tissue under examination to the light source. Consequently, its application in a clinical setting is best suited for intraoperative use to assist in exploring tissue in real time, or via thin fiberoptic needles that can reach the suspected location within the breast for a minimally invasive evaluation.

The breast is complex; its multiple tissue components make it more difficult to classify using optical spectroscopy than other tissues (i.e. esophagus, colon, bladder, or cervix). However, numerous reports over the past ten years highlight a number of spectroscopic approaches capable of detecting cancer and characterizing various types of breast tissue components. Within this set of reports, vibrational spectroscopy has been the method of choice 
for most researchers. This may not be surprising given that the vibrational spectra from breast tissue contain a number of peaks arising from a diverse collection of tissue biomolecules.

\subsection{Vibrational spectroscopy}

Early work indicated that substantial biochemical information is imprinted in the Raman scattering spectra of breast tissues suggesting the potential of this approach for breast cancer diagnosis ${ }^{4-6}$. Redd et al suggested that the Raman spectra from breast tissue specimens contain features that are attributable to various amounts of carotenoids and lipids ${ }^{6}$. A small contribution from a heme-type signal was detected in some samples of benign breast tissue and a much stronger heme-type signal was detected in most of the breast cancers. They also reported that the Raman spectra of diseased breast tissue (benign and malignant) exhibit lower contributions from lipids and reduced contributions from carotenoids.

Frank et al reported through Raman scattering studies with laser wavelengths ranging from 406 to $830 \mathrm{~nm}$ that the best defined lipid features were observed with NIR laser excitation, while carotenoid features were strongest in the blue-green spectral range due to resonance enhancement ${ }^{5}$. Extending their work, this same team later reported that the Raman spectra changed dramatically in diseased specimens, with much less evident lipid bands and the differences between benign (fibrocystic) and malignant lesions were smaller than those between normal and malignant specimens ${ }^{4}$. These observations were later confirmed when Manoharan et al asserted the ability of Raman spectroscopy to accurately classify normal, benign, and malignant breast tissues ${ }^{7}$.

Raman micro-spectroscopy was more recently used to identify the origin of the peaks observed in the vibrational spectrum from breast tissues. Yu et al reported that the spectral 
differences and changes between normal and malignant breast tissue samples could be categorized into the following three groups ${ }^{8}$ : (1) the band from the symmetric stretching modes of the $\mathrm{PO}_{2}^{-}$group in the DNA shifts from 1082 to $1097 \mathrm{~cm}^{-1}$ and becomes stronger; this is accompanied by an increase in the intensity of the symmetric stretching modes of O-P-O at 817 $\mathrm{cm}^{-1}$ in the RNA; (2) the bands of Amide I and III at 1657 and $1273 \mathrm{~cm}^{-1}$ change to 1662 and $1264 \mathrm{~cm}^{-1}$ respectively with their intensity and band-widths also increasing. The peak of the C-O stretching modes in the amino acids shifts to higher wave numbers while the intensity of the tryptophane band at $1368 \mathrm{~cm}^{-1}$ diminishes; (3) fewer Raman bands from lipids are present.

In order to understand the relationship between the Raman spectrum of a sample of breast tissue and its disease state, Shafer-Peltier et al compared NIR Raman spectroscopic images of human breast tissue specimens (acquired using a confocal microscope) with the corresponding hematoxylin- and eosin-stained images ${ }^{9}$. Spectra obtained from the epithelial cell cytoplasm, cell nucleus, fat, beta-carotene, collagen, calcium hydroxyapatite, calcium oxalate dihydrate, cholesterol-like lipid deposits, and water were used to form a basis of breast tissue spectra. By assuming that the macroscopically measured Raman spectra were a linear combination of the basis spectra, they developed a chemical/morphological basis to fit the observed features in normal and diseased breast tissues. This model was used to characterize the composition of ex vivo specimens and pathologies from 57 patients and predict the disease state ${ }^{10}$. The results indicated that the fit coefficients for fat and collagen were the key parameters in the resulting diagnostic algorithm, which classified samples according to their specific pathological diagnoses, attaining 94\% sensitivity and 96\% specificity for distinguishing cancerous tissues from normal and benign tissues. 
The progress to date using Raman spectroscopy to classify breast tissue specimens provides very encouraging results when considered as a tool to evaluate a localized region via statistical means. It is important to mention that preliminary studies using Fourier transform infrared spectroscopy (FTIR) for the analysis of breast specimens yielded similarly promising results ${ }^{11,12}$. Dukor et al reported that benign vs. malignant cells and benign vs. atypical hyperplasia were discriminated with $100 \%$ accuracy, and malignant vs. atypical hyperplasia were discriminated with an accuracy of $90 \%$ and higher ${ }^{12}$. Ci et al reported that the spectral differences observed among normal, benign, and malignant breast tissue samples could be categorized by the following ${ }^{11}$ : (1) the characteristic spectral patterns of fibroadenoma and carcinoma tissues appear in the frequency regions of $950-1150$ and $2800-3050 \mathrm{~cm}^{-1}$ respectively; (2) the peak at $970 \mathrm{~cm}^{-1}$ is sharper and stronger, and the prominent bands at 1204, 1280, and $1338 \mathrm{~cm}^{-1}$ are weaker and broader for carcinoma tissue, whereas the band near $970 \mathrm{~cm}^{-1}$ is weaker and the prominent peaks of collagen are sharper and stronger for benign tissue; (3) the band near $1163 \mathrm{~cm}^{-1}$ in benign tissue shifts to $1171 \mathrm{~cm}^{-1}$ in carcinoma tissue; (4) The absorbance (A) ratios of $\mathrm{A}(1032) / \mathrm{A}(1083)$ and $\mathrm{A}(2958) / \mathrm{A}(2853)$ are the lowest in carcinoma tissue and highest in fibroadenoma tissue; and (5) $\mathrm{A}(1459) / \mathrm{A}(1241)>1.0$ for normal tissue, $\mathrm{A}(1453) / \mathrm{A}(1239) \geq 1.0$ for fibroadenoma, and $\mathrm{A}(1456) / \mathrm{A}(1239) \leq 1.0$ for hyperplasia and carcinoma tissues. $\mathrm{Ci}$ et al concluded that these significant differences revealed the differences in the relative contents of nucleic acids and collagen proteins in breast tissue components. It must be noted that the FTIR measurements discussed above were not obtained directly on the tissue specimens but required extensive tissue processing. Specifically, Ci et al ${ }^{11}$ utilized dehydrated films of cell suspensions extracted from small areas of the specimens. Dukor et al ${ }^{12}$ performed the measurements on the same samples used by pathologists for histopathologic evaluation, e.g. 
stained samples on plain glass slides. This may be a limitation of FTIR spetroscopy that must be resolved prior to its application in a clinical setting.

\subsection{Light scattering methods}

Light scattering spectroscopy methods have been explored by different research groups for their ability to distinguish breast tissue types. Wallon et al measured the NIR reflectance spectra of $1100-2500 \mathrm{~nm}$ in specimens from ten breast cancer patients and found four spectral regions to be different between normal and cancerous tissues: 1208-1242, 1746-1788, 2012-2048 and $2326-2368 \mathrm{~nm}^{13}$. They also reported that even a minute quantity of cancer infiltration could be detected by NIR spectroscopy.

Yang et al observed changes on the absorption spectra in the 250-650 nm range of malignant, fibroadenoma, and normal human breast tissues ${ }^{14-16}$. The absorption spectra were extracted from diffuse reflectance measurements and the main differences in absorption were observed at 255-265 and at 275-285 $\mathrm{nm}$. These differences were attributed to changes in proteins and DNA. A set of critical parameters was proposed for separating malignant tissues from fibroadenoma and normal tissues.

Bigio et al reported on preliminary results of a clinical study designed to test elasticscattering spectroscopy mediated by fiberoptic probes for transdermal-needle (interstitial) measurements (suitable for a minimally invasive diagnosis) and a hand-held probe (suitable for assessing tumor/resection margins during open surgery) ${ }^{17}$. Preliminary results from in vivo measurements on 31 women analyzed using artificial intelligence methods of spectral classification yielded a sensitivity of $69 \%$ and specificity of $85 \%$. The authors suggested that they expected these values to improve with the development of more sophisticated data 
processing algorithms. A fiber-optic needle probe was also used by Van Veen et al to determine the local optical properties of breast tissue in vivo using differential pathlength spectroscopy ${ }^{18}$. This yields information on the local tissue blood content, the local blood oxygenation, the average micro-vessel diameter, the beta-carotene concentration and the scatter slope. The results showed that malignant breast tissue is characterized by a significant decrease in tissue oxygenation and higher blood content compared to normal breast tissue. These in vivo observations may be compared with ex vivo measurements reported by Palmer et al using diffuse reflectance spectroscopy that yielded sensitivity of $30 \%$ and specificity of $78 \%{ }^{19}$. These significant variations in sensitivity as reported by the different groups highlight the importance of developing a common classification algorithm as well as a common measurement scheme of the diagnostic outcome. 

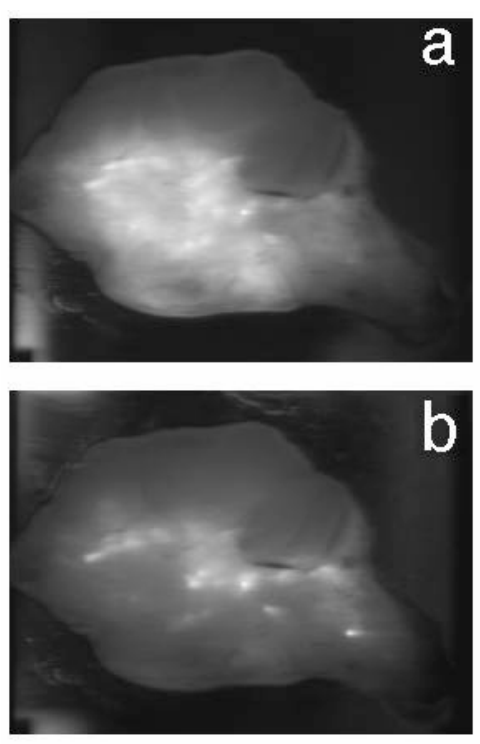
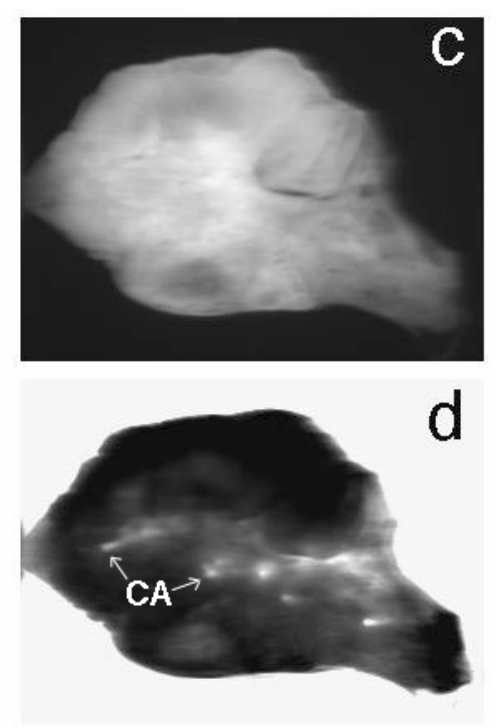
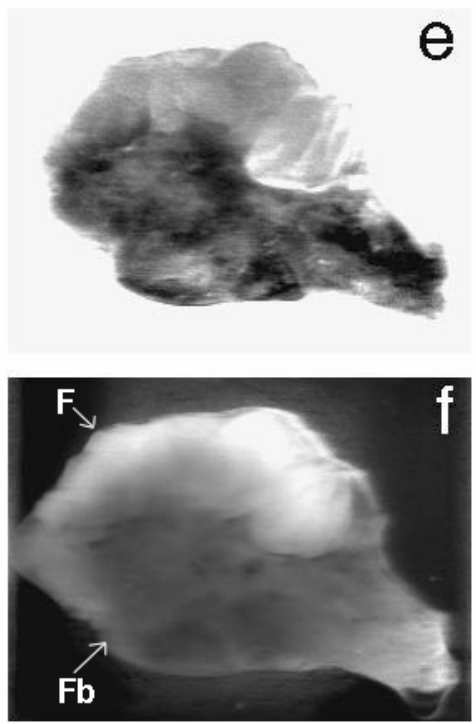

Figure 1: Spectroscopic images of a $\sim 4 \times 3 \mathrm{~cm}^{2}$ human breast tissue, $5 \mathrm{~mm}$ thick, with multifocal high grade ductal carcinoma surrounded by fibrous supporting tissue with an adjacent area of fatty infiltration demonstrating enhanced tissue differentiation using NIR light scattering and autofluorescence approaches. NIR autofluorescence (NIRA) images from 700-1000 nm under laser excitation of a) 532 and b) $633 \mathrm{~nm}$. c) Cross polarized light scattering (CPLS) under 700 $\mathrm{nm}$ illumination. d) Ratio of the $633 \mathrm{~nm}$ NIRA image divided by the $700 \mathrm{~nm}$ CPLS image. e) Inter-image ratio of the CPLS image under $1000 \mathrm{~nm}$ illumination divided by the $700 \mathrm{~nm}$ CPLS image and f) Inter-image ratio of the $700 \mathrm{~nm}$ CPLS image divided by the $532 \mathrm{~nm}$ NIRA image. 


\subsection{Autofluorescence spectroscopy}

Pioneering work by Alfano et al highlighted the potential of autofluorescence spectroscopy for cancer detection ${ }^{20}$. In a paper by Liu et al ${ }^{21}$, Alfano and coworkers explored the use of autofluorescence, Raman scattering and time-resolved light scattering approaches as optical diagnostic techniques to separate diseased and normal breast tissues. Autofluorescence spectroscopic measurements on human breast tissues indicated that the ratio of fluorescence intensity at $340 \mathrm{~nm}$ to that at $440 \mathrm{~nm}$ could be used to distinguish between cancerous and noncancerous tissues. Gupta et al reported on an in vitro study involving 63 patients to evaluate

autofluorescence spectroscopy under excitation in the near-UV region (nitrogen laser) ${ }^{22}$. They reported that significant changes were observed in the spectrally integrated autofluorescence intensity from normal, benign, and cancerous breast tissues. The intensity ratios of cancerous tissues to benign tumor and normal tissues were found to be 3.2 and 2.8 , respectively. A discrimination parameter based on spectrally integrated intensity alone provided a sensitivity and specificity up to $99.6 \%$. A similarly high sensitivity was reported by Hage et al who used laserinduced autofluorescence spectroscopy under $548 \mathrm{~nm}$ excitation ${ }^{23}$.

The experiments in the paper by Palmer et al (discussed in the previous section) included characterization of the tissues using autofluorescence spectroscopy under multiple excitation wavelengths in the ultraviolet-visible range. They were successful in discriminating malignant and nonmalignant tissues with a sensitivity and specificity of $70 \%$ and $92 \%$, respectively ${ }^{19}$. The analysis of the results suggested that the important fluorophores for breast cancer diagnosis are most likely tryptophane, NAD(P)H, and flavoproteins.

Zhang et al investigated the spectral, polarization, and temporal characteristics of the farred and emission beyond $650 \mathrm{~nm}$ of breast tissue specimens under low intensity laser excitation 
at 532 and $633 \mathrm{~nm}^{24}$. The lifetime of this emission was found to be on the order of $1 \mathrm{~ns}$. It was also suggested that the NIR autofluorescence intensity from human breast cancer is different than normal tissue and could potentially be used to detect breast cancer. More recently, Demos et al reported on results from fresh tissue specimens obtained from surgery that were imaged using NIR autofluorescence under 532 and $633 \mathrm{~nm}$ excitation ${ }^{25}$. These images were subsequently compared with the histopathological map of each specimen. The experimental results indicated that the intensity of the NIR emission was considerably different in breast cancer compared to that of the adjacent non-neoplastic tissues (adipose and fibrous tissue). Examples of this approach are shown in Figures 1 and 2. From the images in Figure 1, only the NIR fluorescence image under $632.8 \mathrm{~nm}$ excitation (1b) and the resulting ratio image (1d) demonstrate a correlation with cancer identified by histology with $\sim 1 \mathrm{~mm}$ diameter ductal carcinoma areas appearing as features with higher emission. The ratio image (1d) improves the visibility and contrast of the cancer and provides sharper delineation of the tumor margins. Table 1 summarizes the average values of the ratio of intensities of cancerous to adipose tissue, cancerous to fibrous tissue, and fibrous to adipose tissue. It was suggested that this method may be suitable for real time detection and imaging of breast cancer lesions in a clinically relevant setting. It was also suggested that $632.8 \mathrm{~nm}$ excitation offers key advantages compared to 532 nm excitation for two reasons. First, the images are least affected by blood due to lower absorption. Second, the intensity ratio between fibrous cancer tissue is higher in combination with a lower ratio of fibrous to adipose tissue allows for easier detection (higher contrast) of the cancer in a field of mixed tissue components. 

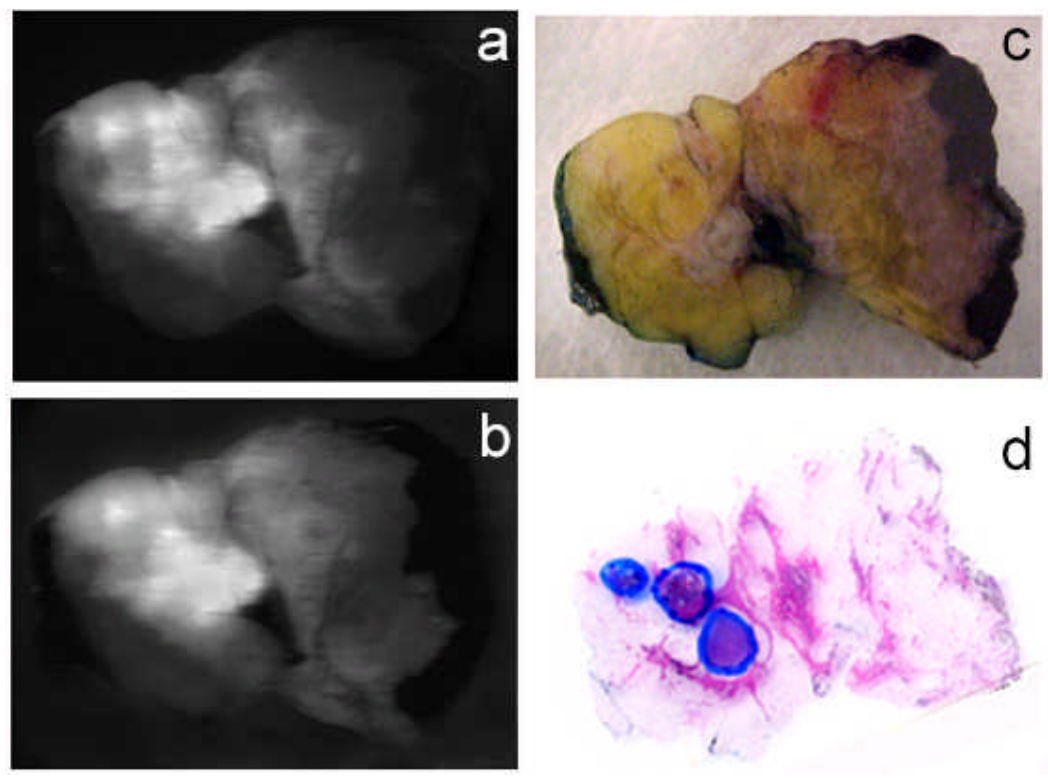

Figure 2: The NIR autofluorescence images of a $\sim 3.2 \times 4.2 \mathrm{~cm}^{2}$ specimen under excitation of a) $532 \mathrm{~nm}$ and b) $632.8 \mathrm{~nm}$. c) A color photograph of the same specimen. d) A contrast-enhanced H\&E stained paraffin section of the same specimen with tumor regions outlined with blue marker.

Table 1: Mean values and standard deviation of the ratio of image intensities from cancer and normal (adipose and fibrous) tissues components as recorded in the NIR autofluorescence images under 532 and $632.8 \mathrm{~nm}$ excitation and in the cross-polarized light scattering images under 700 nm illumination.

\begin{tabular}{|c|c|c|c|}
\hline \multirow{2}{*}{} & \multicolumn{3}{|c|}{ Mean Value / Standard Deviation } \\
\cline { 2 - 4 } & $\begin{array}{c}\mathbf{5 3 2} \mathbf{~ n m} \\
\text { Excitation }\end{array}$ & $\begin{array}{c}\mathbf{6 3 2 . 8} \mathbf{~ n m} \\
\text { Excitation }\end{array}$ & $\begin{array}{c}\mathbf{7 0 0} \mathbf{~ n m} \\
\text { Illumination }\end{array}$ \\
\hline Cander/Adipose & $\mathbf{2 . 6 2} / 0.67$ & $\mathbf{2 . 0 6} / 0.55$ & $\mathbf{1 . 2 1} / 0.17$ \\
\hline Cancer/Fibrous & $\mathbf{1 . 3 4} / 0.11$ & $\mathbf{1 . 4 9} / 0.27$ & $\mathbf{1 . 0 9} / 0.14$ \\
\hline Fibrous /Adipose & $\mathbf{2 . 2 6} / 0.52$ & $\mathbf{1 . 3 0} / 0.12$ & $\mathbf{1 . 1 2} / 0.20$ \\
\hline
\end{tabular}




\subsection{Time-resolved and polarization approaches}

As discussed in the previous section, Liu et al suggested the use of time-resolved light scattering for tissue discrimination ${ }^{21}$. The drawback of this approach is that it requires the use of excised specimens to measure the temporal profiles of ultrashort pulses (on the order of a few picoseconds or less) after propagating through the tissue specimen. This approach was recently employed by Garofalakis et al to retrieve the reduced scattering coefficients of very thin specimens from time-resolved transmission data ${ }^{26}$. Demos et al investigated the degree of polarization of the propagating pulse through normal and cancer breast tissue specimens and demonstrated that the intensity, the polarization state, and the temporal profile of the emerging light were different depending on the tissue type ${ }^{27}$. This change in the polarization state is due to the differences in the scattering properties of the tissues.

The temporal profile and polarization state of the autofluorescence have also been the focus of preliminary studies. Tadrous et al used alcohol-fixed tissue samples photoexcited by pulses at $415 \mathrm{~nm}$ to acquire fluorescence lifetime images that use fluorescence decay differences between tissue components to generate image contrast ${ }^{28}$. Within individual patients there was a statistically significant difference between benign and malignancy-associated stroma. In addition, benign collagen had a longer decay time than benign epithelium. Mohanty et al made steady-state measurements of the anisotropy of autofluorescence from malignant and normal breast tissue specimens and reported a dependence of the anisotropy on tissue thickness and type $^{29}$. 


\subsection{Spectroscopic assessment of lymph nodes and microcalcifications}

The evaluation and treatment of breast cancer requires establishing whether or not the cancer has spread to the lymph nodes. In response to this issue, Raman and light scattering spectroscopy have been explored as methods that may be able to provide rapid, accurate, and straightforward detection of metastases in the lymph nodes. Johnson et al discussed the use of light scattering spectroscopy to interrogate excised nodes with pulsed broadband illumination and collection ${ }^{30}$. The study involved specimens from 68 patients and the analysis of the data suggested $84 \%$ sensitivity and $91 \%$ specificity in detecting the cancerous nodes. Smith et al described the use of Raman scattering spectral mapping in the assessment of axillary lymph

nodes ${ }^{31}$. This method produced false-color spectral images of lymph node sections representing the biochemical composition of heterogeneous lymph node features.

Haka et al demonstrated the use of Raman scattering spectroscopy to characterize microcalcifications in benign and malignant breast lesions ${ }^{32}$. Based on their Raman spectrum, microcalcifications were initially separated into two categories: type I, calcium oxalate dehydrate; and type II, calcium hydroxyapatite. Type I microcalcifications were diagnosed as benign, whereas type II were subdivided into benign and malignant categories using principal component analysis. Using statistical analysis, subtle chemical differences were highlighted in type II that correlated with breast disease. It was suggested that type II microcalcifications that form in benign ducts typically contain a larger amount of calcium carbonate and a smaller amount of protein than those formed in malignant ducts. Using this diagnostic strategy, they were able to distinguish microcalcifications occurring in benign and malignant ducts with a sensitivity of $88 \%$ and a specificity of $93 \%$. 


\section{Chapter 3. Noninvasive optical methods}

\subsection{Background}

Optical spectroscopic interrogation of tissues in vivo can provide information about various indicators of tissue function, thus adding functional information to simple density imaging ${ }^{33,34}$. Studies have indicated that measurements of neovasculature, hypoxia and cellular microenvironments could have a role in tumor detection and treatment planning, as they are all related to tissue function. Hemoglobin is an important parameter because it measures the vascularization of tissue. Since breast cancer tissue is mostly hypoxic because of a metabolic imbalance between oxygen supply and consumption ${ }^{33}$, measuring it optically has significant potential in diagnosis as well as treatment response. The hemoglobin levels measured by NIR imaging for the normal tissue surrounding the tumor may also be representative of the oxygen carrying capacity of the blood in the breast. Pretreatment hemoglobin levels ${ }^{35}$ can also aid in predicting tumor response to primary chemotherapy. Tumor oxygenation may serve to predict its response to radiation treatment as suggested by its critical role in modifying the dose response curve $^{33}$ and may also be related to the likelihood of occurrence of distant metastases ${ }^{36}$. Vaupel et $a{ }^{33}$ have shown that although hypoxia does not correlate with tumor size, location, grade or stage, it depends critically on whole blood hemoglobin levels.

Another parameter of significant interest is scattering. Severe scattering of light as it passes through breast tissue causes a great challenge to optical mammography as it limits the maximum achievable contrast and spatial resolution ${ }^{37-39}$. Optical scattering has been correlated to mammographic density ${ }^{40}$, which is a major risk factor for developing cancer ${ }^{41}$. In breast cancer locations, scattering is certainly expected to increase because the tumor cells stimulate 
endothelial cell proliferation ${ }^{34}$, which increases the cellular density. In addition, the tortuous tumor vessel network is held together by dense fibrotic connective tissue ${ }^{42}$ that may be optically dense, owing to the presence of different size scatterers relative to the surrounding tissue. Spectroscopic studies have shown this increase in scattering in a palpable carcinoma ${ }^{43}$. Recent advances in lasers and opto-electronic instrumentation have opened up the possibility for new methods of overcoming the effects of scatter.

Optical imaging is able to deliver unique spectroscopic information directly related to the physiological status of tissues that is currently not being obtained by other available clinical modalities ${ }^{44}$. However, the turbid nature of tissues prevents embedded inclusions from being spatially resolved with sufficient resolution ${ }^{45}$. Three optical imaging approaches have been developed: time-domain, frequency-domain, and continuous wave (CW). Time-domain optical imaging involves illuminating an object with ultrashort pulses and measuring the temporal profile of the transmitted light. A mathematical model of photon migration is then used to fit all or part of the measured temporal distribution of the transmitted light in a number of geometries. This model is used as if it represents a noise-free estimate of the original data, and images are created with the predicted intensities of transmitted pulses. Frequency-domain techniques involve illuminating an object with an intensity-modulated beam, and measuring the $\mathrm{AC}$ modulation amplitude and phase shift of the transmitted signal, typically using a standard heterodyne method. The transport of the modulated beam is often described in terms of the propagation of so-called photon density waves. CW optical imaging systems require a source that either emits at a constant intensity, or is modulated at a low frequency (a few $\mathrm{kHz}$ ) in order to exploit the significant improvements in sensitivity available from phase-locked detection techniques. Measurements of the intensity of light transmitted between two points on the surface 
of tissue are not only relatively straightforward and inexpensive to obtain, but also contain a remarkable amount of useful information.

\subsection{NIR spectroscopy and imaging}

Conover et al acquired NIR spectroscopic measurements in vivo on subcutaneous rat mammary adenocarcinomas and showed through sensitivity and specificity analyses that NIR spectroscopy imaging may identify clinically relevant hypoxia, even when its spatial extent is below the resolution limit of the NIR spectroscopy technique ${ }^{46}$.

Tromberg et al use a handheld frequency-domain system and observed a decrease in oxygen saturation in spectroscopic studies on a palpable mass diagnosed as ductal carcinoma in $s i t u^{43}$. In a related study that monitored neoadjuvant chemotherapy using spectroscopy, initial tissue oxygen saturation was lower in the lesion with respect to the surrounding tissue followed by a slight peak, when tracked over 10 weeks of treatment ${ }^{47}$.

Chance et al developed a NIR method capable of rapidly acquiring data from the breast with a handheld puck ${ }^{48}$. The $\mathrm{CW}$ device was used to measure tumor hemoglobin and hemoglobin desaturation compared to the mirror image site on the contralateral normal breast. Results found an increased hemoglobin concentration due to angiogenesis and decreased hemoglobin saturation due to hyper-metabolism of the cancer in the cancerous breast compared to the normal breast. Receiver Operating Characteristic (ROC) evaluation of the nomogram showed $95 \%$ in the area under the curve (AUC) for 116 patients with tumor sizes down to and including those of $0.8-1 \mathrm{~cm}$ in diameter.

Simick et al used NIR transillumination spectroscopy to show that optical spectroscopy predicted the radiological assessment of density with a principal component analysis model in 
the range of $90 \%$ with an odds ratio comparable to mammography ${ }^{49}$. Parameters such as oxygen saturation, water fraction, and scattering provided fundamental metabolic information about tissue that is not currently being obtained with any other imaging modality.

Rinneberg et al recorded optical projection mammograms at two wavelengths (670 and $785 \mathrm{~nm}$ ) using a scanning time-domain instrument ${ }^{50}$. From distributions of times of flight of photons through the slightly compressed breast sampled at a large number of scan positions, optical mammograms were generated by various methods of data analysis. In a study of 102 histologically confirmed carcinomas of 154 patients, 72 carcinomas were detected retrospectively in both craniocaudal and mediolateral projections of the tumor-bearing breast, an additional 20 cases in one projection only, while 10 carcinomas were missed altogether in optical mammograms. Optical properties of each of the 87 tumors identified in optical mammograms were analyzed using the diffraction model of photon density waves, including in the analysis all prior knowledge available. From absorption coefficients at 670 and $785 \mathrm{~nm}$, physiological parameters were deduced. Apart from a few exceptions, they found that the total hemoglobin concentration of tumors exceeded that of healthy breast tissue, whereas blood oxygen saturation was a poor discriminator by itself.

\subsection{Optical tomography}

3D optical tomography utilizes localized tissue optical properties which are converted into functional parameters, such as concentrations of oxy- and deoxy-hemoglobin, blood volume, and blood oxygenation. An ability to derive such parameters from optical images of the breast has already been demonstrated ${ }^{51,52}$. Past approaches of NIR tomography have suffered from using too few wavelengths and simplistic strategies for spectral deconvolution. More recently 
there have been significant breakthroughs in the development of algorithms that incorporate multispectral information associated with the different chromophore-specific absorption spectra into the image reconstruction process ${ }^{39,40,53}$.

Many theoretical constructs have been proposed to analyze experimental data such as using different photon time-of-flight or frequency intervals to separate the effects of scattering and absorption. This enables one to quantify optical coefficients at different wavelengths as a spectroscopic signature of abnormal tissue embedded in thick, but otherwise normal tissue. The most widely used of these theoretical constructs are diffusion-like models based on the diffusion approximation of the transport equation and models based on the random walk theory (RWT) ${ }^{54}$. These theories can be used to fit experimental data and retrieve optical properties.

Methods currently being pursued generally involve either compressing the breast between two parallel arrays of sources and detectors (or between plates over which individual sources and detectors are scanned) ${ }^{55-59}$, or coupling sources and detectors in one or more rings around the surface of the uncompressed breast ${ }^{37,60,61}$. The former approach is particularly appropriate for generating single projection images, while the latter is more readily able to yield depth information sufficient for a full 3D image reconstruction.

The effect of an inclusion on photon propagation is usually considered either under a perturbation approximation that involves serious restrictions on the inclusion characteristics, or in the frames of RWT, where the influence of increased scattering is modeled by a photon time delay (random walker) proportional to a squared size of the inclusion ${ }^{62}$. Analysis of absorptive abnormalities is expanded beyond the small linear perturbations to the case of larger nonlocalized targets, by introducing an exponential correction factor that depends on the inclusion size and magnitude of absorption perturbation ${ }^{62,63}$. 
Time-resolved data have been obtained from an optical mammograph designed by researchers at the Physikalich.-Techniche-Bundesanstalt (PTB) of Berlin ${ }^{56,64,65}$ (described in the previous section) of several patients with invasive ductal carcinoma (IDC). The data were used by Gandjbakhche et al to estimate optical coefficients (absorption and scattering) of the lesion and surrounding normal tissue. Then, physiologically important tissue characteristics, i.e., total blood volume (TBV) and blood oxygenation $\left(\mathrm{S}_{\mathrm{O} 2}\right)$ were obtained from relative concentrations of oxy- and deoxy-hemoglobin, using their known spectral absorptions with ad hoc assumptions of constant water content of $30 \%$ and an insignificant role of lipids in the tissue absorption at these wavelengths. In some (but not all) cases, the tumors can be seen in the optical images, corresponding to both projections, as illustrated in Figure 3.

Table 2 shows calculated ratios of total blood volume and blood oxygenation of tumors relative to surrounding normal tissue for three patients. Though the statistics are small, these first results give evidence that the malignant tissue in the IDC is hypoxic (in a deoxygenated state) with considerably higher blood volume compared to the surrounding tissue. The latter can be explained by increased vascularization. 

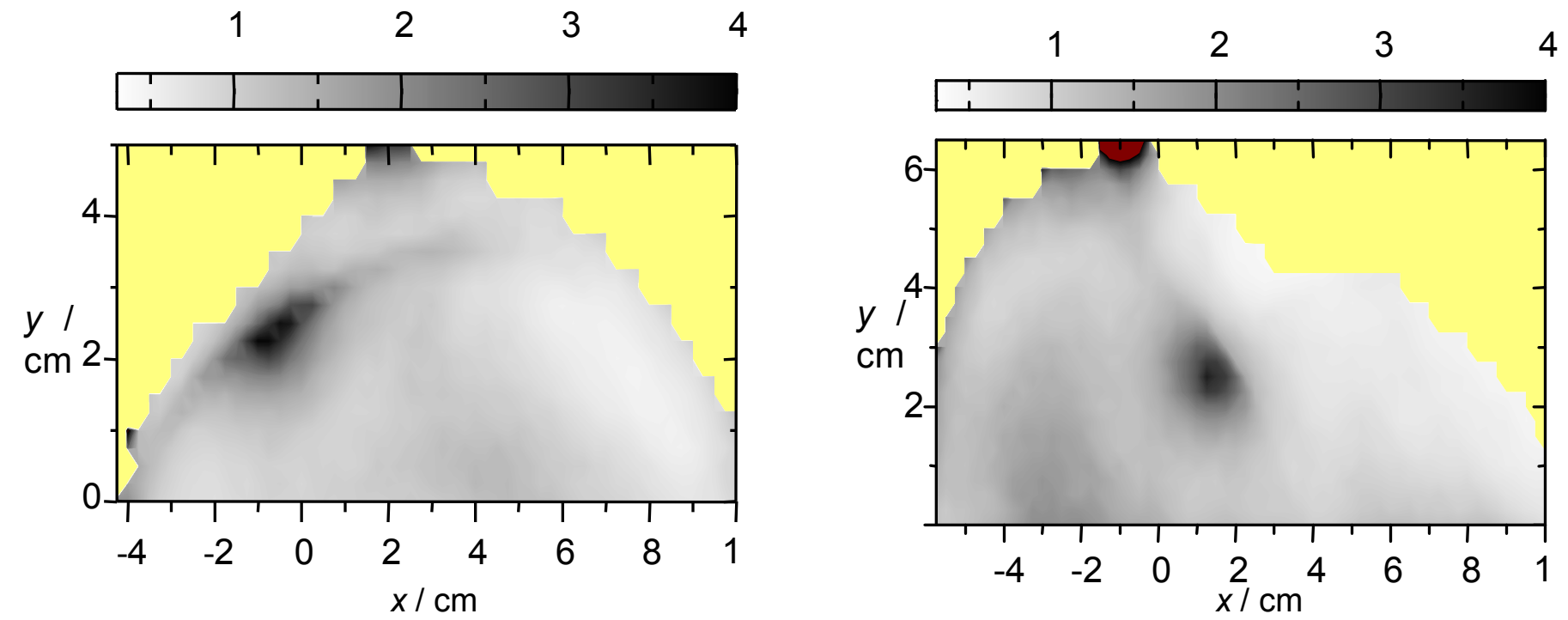

Figure 3: 2D optical images of the breast, bearing IDC at different projections: a) craniocaudal and b) mediolateral.

Table 2: Calculated ratios of total blood volume and blood oxygenation for three subjects

\begin{tabular}{|l|l|l|l|}
\hline Age & Tumor Size (mm) & TBV(tumor)/TBV(tissue) & So2 $_{\text {o } \text { (tumor)/So }}$ (tissue) \\
\hline 84 & $25 \times 15 \times 15$ & 1.8 & 0.77 \\
\hline 57 & $40 \times 35 \times 30$ & 2.0 & 0.90 \\
\hline 52 & $15 \times 15 \times 15$ & 2.1 & 0.80 \\
\hline
\end{tabular}


In previous work at Dartmouth College, McBride et al ${ }^{66}$ used a frequency-domain NIR tomography system to image a subject with a $2.5 \mathrm{~cm}$ IDC, and although an increase in hemoglobin was observed, a reduction in oxygen saturation was not found. Dehghani et al ${ }^{40}$ used 3D modeling to obtain tomographic images from a patient with IDC and reported an increase in blood oxygen saturation level, contrary to expectation.

In a study using time-domain instrumentation with two wavelengths and a water contribution fixed at 30\%, lower oxygen saturation was observed in images of two carcinomas ${ }^{63}$. Heffer et al ${ }^{58}$ used an 'oxygenation index' generated from frequency-domain measurements to show a decrease in carcinomas.

Yates et al from University College London developed a time-resolved optical tomography system to generate cross-sectional images of the human breast ${ }^{67}$. The 3D breast imaging scheme was based on a 32-channel time-domain imaging system and recorded the temporal distribution of transmitted light at up to 32 locations on the surface simultaneously in response to illumination by picosecond pulses of light at wavelengths of 780 and $815 \mathrm{~nm}{ }^{68}$. Images were generated using a reconstruction package known as TOAST (temporal optical absorption and scattering tomography), which determines the optical parameters that describe a finite-element model (FEM) of photon migration within an object by comparing its predictions with the measured data ${ }^{69}$. The parameters of the model were then adjusted iteratively to minimize the difference between the two, and thereby 3D images were produced that represent the internal distribution of scatter and absorption ${ }^{70}$. Thirty-eight studies have been performed on 3 healthy volunteers and 21 patients with a variety of breast lesions including cancer. 17 out of 19 lesions were successfully detected, and optical images of the healthy breast of the same volunteer displayed a heterogeneity that was repeatable over a period of months. 
Srinivasan ${ }^{71}$ used multi-wavelength NIR tomography to noninvasively quantify physiological parameters of breast tumors using direct spectral reconstruction. Frequency domain NIR measurements were incorporated with a new spectrally constrained direct chromophore and scattering image reconstruction algorithm, which was validated in simulations and experimental phantoms. Images of total hemoglobin, oxygen saturation, water and scatter were obtained with higher accuracy than previously reported. Three of six cases studied were malignant (infiltrating ductal carcinomas) and showed higher hemoglobin (34-86\%), a reduction in oxygen saturation, an increase in water content as well as scatter changes relative to surrounding normal tissue. Three of the six cases were benign, two of which were diagnosed with fibrocystic disease and showed a dominant contrast in water, consistent with fluid-filled cysts. Scatter amplitude was the main source of contrast in the volunteer with the benign fibrosis, which typically contains denser collagen tissue. The changes monitored correspond to physiological changes associated with angiogenesis, hypoxia, and cell proliferation anticipated in cancers. These changes represent potential diagnostic indicators, which can be assessed to characterize breast tumors. The physiological changes observed can be extended to monitoring response to therapy, and predicting risk of malignancy or aggressiveness of tumors. NIR tomography is an imaging method that can directly quantify some functional processes noninvasively.

While clinical data are useful to identify overall trends separating malignant from benign disease, the reality may be that separating the two is more complicated. Certain features of benign conditions, such as fibrocystic disease, present difficulties in diagnosing it from cancer, when based only upon mammographic image features ${ }^{42}$. NIR tomography is undergoing several 
clinical trials to evaluate its role in aiding breast lesion diagnosis ${ }^{43}, 72$; however, defining the particular NIR characteristics expected in different types of tumors is not yet well defined.

\subsection{Multimodality techniques}

Since the spatial resolution of optical tomography is limited by scattering, multi-modality imaging is increasingly being used to interrogate tissue morphology and function simultaneously because of the inherently optimized co-registration. Structure and function of tissue afford different physical bases for contrast and combinations have proven beneficial in the diagnosis and management of disease ${ }^{73}$.

To date, NIR techniques have been combined with several high spatial resolution, structure-specific imaging modalities including X-ray tomosynthesis ${ }^{57}$, ultrasound ${ }^{66,74}$, and MRI ${ }^{75-77}$, to study human tissues and small animals. Past experiences have contributed to a variety of imaging systems, imaging geometries, and numerical reconstruction techniques, but have not led to a consensus on the optimal way of applying a priori derived constraints.

Adjunctive noninvasive imaging modalities are often needed to characterize suspicious abnormalities, especially in women with radiographically dense breasts. There is considerable potential for functional NIR imaging to help distinguish breast cancer noninvasively, yet this modality has consistently suffered from low spatial resolution. Such hybrid approaches could generate image data that combine the functional information of optical imaging with the high spatial resolution of structural information in MRI. Hybrid imaging systems also avoid complications associated with tissue movement between separate exams, which reduce coregistration accuracy and thus degrade the diagnostic value of the image fusion ${ }^{78}$. 
In addition to co-registration, data sets from combined NIR and MRI imaging offer other synergistic benefits, namely anatomical priors (from high spatial resolution MRI) enhance NIR (i.e. high contrast resolution) image reconstruction. NIR spectroscopy is biochemically rich, but spectroscopic imaging is hindered by the highly scattered photon paths that reduce resolution in tissue $^{51}$. The most widely adopted approach to this problem incorporates parameter estimation strategies based on models of light propagation in tissue. The estimation task is sensitive to small perturbations in the light measurements, not all of which are caused by the intrinsic changes in tissue optical properties. Experience has shown that significant improvements in the stability and accuracy of the reconstruction process can be obtained by including prior anatomical/optical information $^{53,57,60,79-81}$.

Techniques for incorporating this information are relatively new, and are the subject of active research in a variety of disciplines, including medical imaging ${ }^{76,82,83}$, industrial process imaging ${ }^{84}$, and geophysical surveying ${ }^{85}$, yet there is no clear consensus on the optimal approach.

Brooksby et al ${ }^{86}$ used NIR tomography and magnetic resonance imaging (MRI) on a healthy woman in vivo. The NIR image reconstruction technique implemented the MR structure as a soft-constraint in the NIR property estimation. The algorithm incorporated the MR spatially segmented regions into a regularization matrix that linked locations with similar MR properties. This reconstruction algorithm allowed maximal flexibility upon use with a priori data, and was validated on a series of breast tissue-like phantoms. Spatially resolved images of absorption and reduced scattering coefficients at multiple wavelengths $(660-850 \mathrm{~nm})$ were used to estimate values of hemoglobin concentration, oxygen saturation, water fraction, scattering power, and scattering amplitude within adipose and glandular breast tissue types identified from MRI. 
Dramatic changes in spatial property distributions arose when the MRI was used to guide these reconstructions. Glandular tissue was observed to have more than four times the water than adipose tissue, and almost twice as much blood volume, as well as slightly reduced oxygen saturation.

Hsiang et al ${ }^{87}$ developed a handheld scanning probe based on broadband diffuse optical spectroscopy (DOS) in combination with dynamic contrast enhanced MRI (DCE-MRI) to quantitatively characterize locally-advanced breast cancers in six patients. Measurements were performed sequentially using external fiducial markers for co-registration. Lesion patterns were categorized according to MRI morphological data, and 3D DCE-MRI slices were converted into a volumetric matrix with isotropic voxels to generate views that coincided with the DOS scanning plane. Lesion volume and depth at each DOS measurement site were determined, and a tissue optical index (TOI) that reflects both angiogenic and stromal characteristics was derived from broadband DOS data. 
(a)
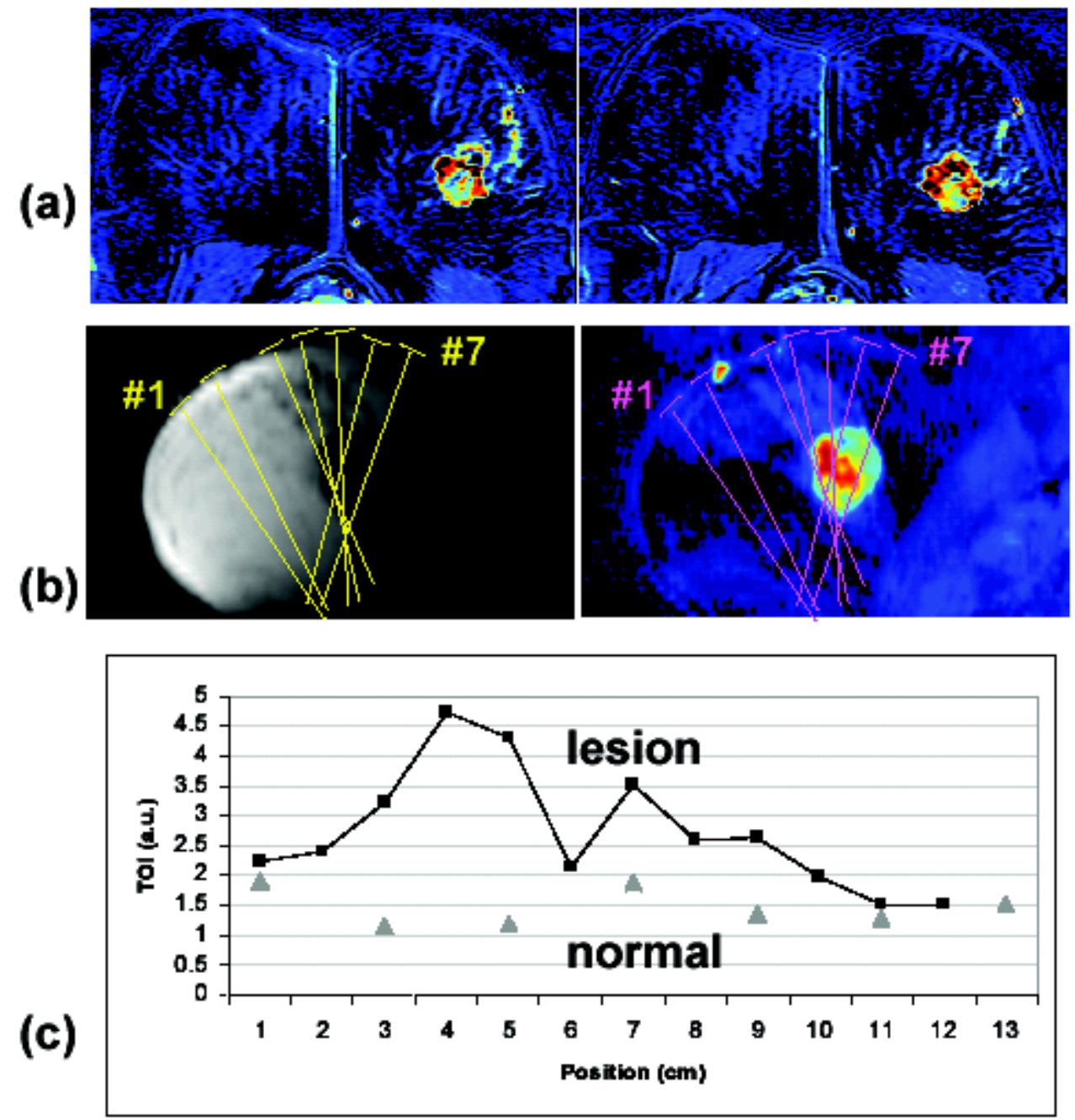

Figure 3: a) The original enhancement maps from 2 slices (4 mm thick) demonstrates an unenhanced necrotic center in the lesion. b) The re-sliced pre-contrast image along the 7 DOS markers demonstrating a hypointense mass, and the corresponding enhancement map demonstrating a strongly enhanced mass. These images are $1.5 \mathrm{~cm}$ thick, and do not show the necrotic core. c) The tissue optical index curve measured at each DOS location from the lesion and the normal breast tissues in the contralateral breast. A dip is seen at $6 \mathrm{~cm}$ in the DOS scan line, likely due to the necrotic core. 


\section{Chapter 4. Discussion}

The histological examination of breast tissue is currently performed by selecting one of the well-developed invasive breast biopsy techniques (i.e. excisional biopsy, axillary node dissection, sentinel node dissection, or fine needle aspiration) depending on the location, size, palpability, and characteristics of the abnormality. Breast excisions remain one of the most common surgical operations for diagnosing and treating breast cancer. In the current setting, while frozen section analysis is available, there are technical limitations to cutting certain types of tissue and as a result, immediate histological analysis is not possible or practical. Therefore, developing technology that can offer detection and delineation of tumor margins in real time may be very useful to a surgeon during a diagnostic or therapeutic procedure.

The progress to date in using various optical spectroscopy methods for the classification of breast tissue arguably provides a solid foundation for the development of spectroscopy-based instrumentation for real time pathological assessment. Most of the approaches use single point measurement techniques that interrogate a small volume of tissue at each measurement. As mentioned earlier, the potential role of this technology in addressing clinical needs related to breast cancer detection and treatment may be for intraoperative tissue characterization in real time, or via designing thin fiberoptic needles to reach the suspected location within the breast for an evaluation of a suspected lesion. Such "optical needle biopsies" have recently been designed and preliminary in vivo results are encouraging ${ }^{17,88}$. Similar work is also in progress by other research teams ${ }^{89}$.

Point spectroscopic measurements or spectral imaging may be used to detect a tumor or establish tumor margins in real time in the operating room. It is estimated that $40-70 \%$ of 
margins from breast lumpectomies will be involved by malignancy if the excision was not preceded by a tissue diagnosis. Optical biopsy methods could be most useful to detect nonpalpable or very small lesions and therefore guide the surgeon.

Although the development of optical mammography as a means of routine screening for early incidence of breast cancer in asymptomatic women is not universally regarded as an achievable goal, several applications have been identified for which the technique may be beneficial for specific patients. It has recently been proposed that NIR imaging may be useful for prescreening younger women to identify those at increased risk of developing disease ${ }^{51}$. The facility to study tissue function, such as the oxygenation status of hemoglobin, may also make

optical imaging a potent tool for detecting responses to new and existing forms of therapy ${ }^{47}$. A further potential application of NIR imaging is to assess and monitor surrounding healthy tissues after treatment.

\section{Acknowledgements}

The authors acknowledge the following agencies for support of their research in breast cancer: The California Breast Cancer Research Program and the Center for Biophotonics, an NSF Science and Technology Center, managed by the University of California, Davis, under Cooperative Agreement No. PHY 0120999. This work was performed in part at UC, Lawrence Livermore National Laboratory under the auspices of the U.S. Department of Energy under Contract W-7405-Eng-48. This work was supported by the Intramural Program of the National Institutes of Health, National Institute of Child Health and Human Development. 


\section{References}

1. Carney PA, Kasales CJ, Tosteson ANA, Weiss JE, Goodrich ME, Poplack SP, Wells WS, Titus-Ernstoff L: Likelihood of additional work-up among women undergoing routine screening mammography: the impact of age, breast density, and hormone therapy use, Preventive Medicine 2004, 39:48-55

2. Hindle WH, Davis L, D W: Clinical value of mammography for symptomatic women 35 years of age and younger., Am J Obstet Gynecol 1999, 180:1484-1490

3. Hata T, H. Takahashi, K. Watanabe, M. Takahashi, K. Taguchi, T. Itoh, and S. Todo: Magnetic Resonance Imaging for Preoperative Evaluation of Breast Cancer: A Comparative Study with Mammography and Ultrasonography, J. Am. Coll. Surg. 2004, 198:190-197

4. Frank C, McCreery R, Redd D: Raman-spectroscopy of normal and diseased human breast tissues, Anal Chem 1995, 67:777-783

5. Frank C, Redd D, Gansler T, McCreery R: Characterization of human breast specimens with Near-IR Ramam spectroscopy, Anal Chem 1994, 66:319-326

6. Redd D, Feng Z, Yue K, Gansler T: Raman-Spectroscopic Characterization of Human Breast Tissues - Implications for Breast-Cancer Diagnosis, Applied Spectroscopy 1993, 46:787-791

7. Manoharan R, Shafer K, Perelman L, Wu J, Chen K, Deinum G, Fitzmaurice M, Myles J, Crowe J, Dasari R, Feld M: Raman spectroscopy and fluorescence photon migration for breast cancer diagnosis and imaging, Photochem. Photobiol. 1998, 67:15-22

8. Yu G, Xu X, Niu Y, Wang B, Song Z, Zhang C: Studies on human breast cancer tissues with Raman microspectroscopy, Spectroscopy and Spectral Analysis 2004, 24:1359-1362

9. Shafer-Peltier K, Haka A, Fitzmaurice M, Crowe J, Myles J, Dasari R, Feld M: Raman microspectroscopic model of human breast tissue: implications for breast cancer diagnosis in vivo, J. Raman Spectroscopy 2002, 33:552-563

10. Haka A, Shafer-Peltier K, Fitzmaurice M, Crowe J, Dasari R, Feld M: Diagnosing breast cancer by using Raman spectroscopy, PNAS 2005, 102:12371-12376

11. Ci Y, Gao T, Feng J, Guo Z: Fourier transform infrared spectroscopic characterization of human breast tissue: Implications for breast cancer diagnosis, Applied Spectroscopy 1999, 53:312-315

12. Dukor R, Liebman M, Johnson B: A new, non-destructive method for analysis of clinical samples with FT-IR microspectroscopy. Breast cancer tissue as an example, Cellular and Molecular Biology 1998, 44:211-217

13. Wallon J, Yan S, Tong J, Meurens M, Haot J: Identification of breast carcinomatous tissue by near-infrared reflectance spectrocsopy, Applied Spectroscopy 1994, 48:190-193

14. Yang Y, Celmer E, Koutcher J, Alfano R: UV reflectance spectroscopy probes DNA and protein changes in human breast tissues, J. Clinical Laser Medicine \& Surgery 2001, 19:35-39

15. Yang Y, Celmer E, Koutcher J, Alfano RR: DNA and protein changes caused by disease in human breast tissues probed by the Kubelka-Munk spectral function, Photochem. Photobiol. 2002, 75:627-632

16. Yang Y, Katz A, Celmer E, Zurawska-Szczepaniak M, Alfano R: Fundamental differences of excitation spectrum between malignant and benign breast tissues, Photochem. Photobiol. 1997, 66:518-522 
17. Bigio I, Bown S, Briggs G, Kelley C, Lakhani S, Pickard D, Ripley P, Rose I, Saunders C: Diagnosis of breast cancer using elastic-scattering spectroscopy: preliminary clinical results, J. Biomed Opt. 2000, 5:221-228

18. Van Veen R, A A, Menke-Pluymers M, Van der Pol C, Sterenborg HJCM: Optical biopsy of breast tissue using differential path-length spectroscopy, Phys. Med. Biol. 2005, 50:2573-2581

19. Palmer G, Zhu C, Breslin T, Xu F, Gilchrist K, Ramanujam N: Comparison of multiexcitation fluorescence and diffuse reflectancy spectroscopy for the diagnosis of breast cancer, IEEE Trans Biomed Eng 2003, 50:1233-1242

20. Alfano RR, Tata D, Cordero J, Tomashefsky P, Longo F, Alfano M: Laser-induced fluorescence spectroscopy from native cancerous and normal tissue, IEEE J. Quantum Electronics 1984, 20:1507-1511

21. Liu C, Das B, Glassman W, Tang G, Yoo KM, Zhu H, Akins D, Lubicz S, Cleary J, Prudente R, Celmer E, Caron A, Alfano R: Raman, fluorescence, and time-resolved lightscattering as optical diagnostic-techniques to separate diseased and normal biomedical media, J. Photochemistry and Photobiology B-Biology 1992, 16:187-209

22. Gupta P, Majumder S, Uppal A: Breast cancer diagnosis using N2 laser excited autofluorescence spectroscopy, Lasers in Surgery and Medicine 1997, 21:417-422

23. Hage R, Galhanone P, Zangaro R, Rodrigues K, Pacheco M, Martin A, Netto M, Soares F, da Cunha I: Using the laser-induced fluorescence spectroscopy in the differentiation between normal and neoplastic human breast tissue, Lasers in Medical Science 2003, 18:171-176

24. Zhang G, Demos S, Alfano R: Far-red and NIR spectral wing emission from tissues under $532 \mathrm{~nm}$ and $632 \mathrm{~nm}$ photo-excitation, Lasers in the Life Sciences 1999, 9:1-16

25. Demos S, Bold R, deVere White R, Ramsamooj R: Investigation of near infrared autofluorescence imaging for the detection of breast cancer, J. Selected Topics in Quantum Electronics In press,

26. Garofalakis A, Zacharakis G, Filippidis G, Sanidas E, Tsiftsis D, Stathopoulos E, Kafousi M, Ripoll J, Papazoglou T: Optical characterization of thin femal breast biopsies based on the reduced scattering coefficient, Phys. Med. Biol. 2005, 50:2583-2596

27. Demos S, Savage H, Heerdt A, Schantz S, Alfano R: Time Resolved Degree of Polarization for Human Breast Tissue, Optics Communications 1996, 124:439-442

28. Tadrous P, Siegel J, French PMW, Shousha S, Lalani E, Stamp G: Fluorescence lifetime imaging of unstained tissues: early results in human breast cancer, J. Pathology 2003, 199:309-317

29. Mohanty SG, N, Majumder S, Gupta P: Depolarization of autofluorescence from malignant and normal human breast tissues, Appl Opt 2001, 40:1147-1154

30. Johnson K, Chicken DW, Pickard D, Lee A, Briggs G, Falzon M, Bigio I, Keshtgar MRS, Bown S: Elastic scattering spectroscopy for intraoperative determination of sentinel lymph node status in the breast, J. Biomed Opt. 2004, 9:1122-1128

31. Smith J, Kendall C, Sammon A, Christie-Brown J, Stone N: Raman spectral mapping in the assessment of axillary lymph nodes in breast cancer, TCRT 2003, 2:327-331

32. Haka A, Shafer-Peltier K, Fitzmaurice M, Crowe J, Dasari R, Feld MS: Identifying microcalcifications in benign and malignant breast lesions by probing differences in their chemical composition using Raman spectroscopy, Cancer Res 2002, 62:5375-5380 
33. Vaupel P, Kallinowski F, Okunieff P: Blood Flow, Oxygen and Nutrient Supply, and Metabolic Microenvironment of Human Tumors : A Review., Cancer Research 1989, 49:6449-6465

34. Kuszyk BS, Corl FM, Franano FN, Bluemke DA, Hofmann LV, Fortman BJ, Fishman EK: Tumor transport physiology: implications for imaging and image-guided therapy, AJR 2001, 177:747-753

35. Bottini A, Berruti A, Brizzi MP, Bersiga A, Generali D, Allevi G, Aguggini S, Bolsi G, Bonardi S, Bertoli G, Alquati P, Dogliotti L: Pretreatment haemoglobin levels significantly predict the tumour response to primary chemotherapy in human breast cancer, $\mathrm{Br} \mathrm{J}$ Cancer 2003, 89:977-982

36. Brizel DM, Scully SP, Harrelson JM, Layfield LJ, Bean JM, Prosnitz LR, Dewhirst MW: Tumor Oxygenation Predicts for the Likelihood of Distant Metastases in Human Soft Tissue Sarcoma, Cancer Research 1996, 56:941-943

37. Dehghani H, Pogue BW, Poplack SP, Paulsen KD: Multiwavelength three-dimensional near-infrared tomography of the breast: initial simulation, phantom, and clinical results, Applied Optics 2003, 42:135-145

38. Hattery D, Chernomordik V, Loew M, Gannot I, Gandjbakhche AH: Analytical solutions for time-resolved fluorescence lifetime imaging in a turbid medium such as tissue, JOSA(A) 2001, 18:1523

39. Li A, Zhang Q, Culver JP, Miller EL, Boas DA: Reconstructing chromosphere concentration images directly by continuous-wave diffuse optical tomography, Optics Letters 2004, 29:256-258

40. Srinivasan S, Pogue BW, Jiang S, Dehghani H, Kogel C, Soho S, Gibson JJ, Tosteson TD, Poplack SP, Paulsen KD: Interpreting hemoglobin and water concentration, oxygen saturation, and scattering measured in vivo by near-infrared breast tomography, Proc. Nat. Acad. Sci. 2003, 100:12349-12354

41. Mandelson MT, Oestreicher N, Porter PL, White D, Finder CA, Taplin SH, White E: Breast density as a predictor of mammographic detection: comparison of interval- and screen-detected cancers, J Natl Cancer Inst 2000, 92:1081-1087

42. Thomsen S, Tatman D: Physiological and Pathological Factors of Human Breast Disease That can Influence Optical Diagnosis, Ann. N. Y. Acad. Sci. 1998, 838:171-193

43. Tromberg BJ, Shah N, Lanning R, Cerussi A, Espinoza J, Pham T, Svaasand L, Butler J: Non-invasive in vivo characterization of breast tumors using photon migration spectroscopy, Neoplasia 2000, 2:26-40

44. Gandjbakhche A: Diffused Optical Imaging and spectroscopy, in vivo, C. R. Academy of Science Paris 2001, 2, series IV:1073-1079

45. Gandjbakhche AH, Nossal R, Bonner RF: Resolution limits for optical transillumination of abnormalities deeply embedded in tissues, Med. Phys. 1994, 21:185-191

46. Conover DL, Fenton BM, Foster TH, Hull EL: An evaluation of near infrared spectroscopy and cryospectrophotometry estimates of haemoglobin oxygen saturation in a rodent mammary tumour model, Physics in Medicine \& Biology 2000, 45:2685-2700

47. Jakubowski DB, Cerussi AE, Bevilacqua F, Shah N, Hsiang D, Butler J, Tromberg BJ: Monitoring neoadjuvant chemotherapy in breast cancer using quantitative diffuse optical spectroscopy: a case study, J Biomed Opt 2004, 9:230-238

48. Chance B, Nioka S, Zhang J, Conant EF, Hwang E, Briest S, Orel SG, Schnall MD, Brian J. Czerniecki V, No, August Breast Cancer Detection Based on Incremental Biochemical 
and Physiological Properties of Breast Cancers: A Six-Year, Two-Site Study, Academic Radiology 2005, 12:925-933

49. Simick MK, Jong R, Wilson B, Lilge L: Non-ionizing near-infrared radiation transillumination spectroscopy for breast tissue density and assessment of breast cancer risk, Journal of Biomed Opt 2004, 9:794-803

50. Rinneberg H, Grosenick D, Moesta KT, Mucke J, Gebauer B, Stroszczynski C, Wabnitz H, Moeller M, Wassermann B, Schlag PM: Scanning Time-domain Optical Mammography: Detection and Characterization of Breast Tumors in vivo, TCRT 2005, 4:483-496

51. Pogue B, Jiang S, Dehghani H, Kogel C, Soho S, Srinivasan S, Song X, Tosteson T, Poplack S, Paulsen K: Characterization of hemoglobin, water, and NIR scattering in breast tissue: analysis of intersubject variability and menstrual cycle changes, J Biomed. Opt. 2004, 9:541-552

52. Torricelli A, Spinelli L, Pifferi A, Taroni P, Cubeddu R, Danesini G: Use of a nonlinear perturbation approach for in vivo breast lesion characterization by multi-wavelength timeresolved optical mammography, Opt. Express 2003, 11:853-967

53. Corlu A, Choe R, Durduran T, Lee K, Schweiger M, Arridge SR, Hillman EM, Yodh AG: Diffuse optical tomography with spectral constraints and wavelength optimization, Appl Opt 2005, 44:2082-2093

54. Hebden JC, Yates TD, Gibson A, Everdell N, Arridge SR, Chicken DW, Douek M, Keshtgar MRS: Monitoring recovery after laser surgery of the breast with optical tomography: a case study, Applied Optics 2005, 44:1898-1904

55. Culver JP, Choe R, Holboke MJ, Zubkov L, Durduran T, Slemp A, Ntziachristos V, Chance B, Yodh AG: Three-dimensional diffuse optical tomography in the parallel plane transmission geometry: evaluation of a hybrid frequency domain/continuous wave clinical system for breast imaging., Med. Phys. 2003, 30:235-247

56. Grosenick D, Moesta KT, Wabnitz H, Mucke J, Stroszczynski C, Macdonald R, Schlag PM, Rinneberg H: Time-domain optical mammography: initial clinical results on detection and characterization of breast tumors, Appl. Opt. 2003, 42:170-186

57. Li A, Miller EL, Kilmer ME, Brukilacchio TJ, Chaves T, Stott J, Zhang Q, Wu T, Chorlton M, Moore RH, Kopans DB, Boas DA: Tomographic optical breast imaging guided by three-dimensional mammography, Appl. Opt. 2003, 42:5181-5190

58. Pera VE, Heffer EL, Siebold H, Schütz O, Heywang-Köbrunner S, Götz L, Heinig A, Fantini S: Spatial secondderivative image processing: an application to optical mammography to enhance the detection of breast tumors, J. Biomed. Opt. 2003, 8:517-524

59. Taroni P, Danesini G, Torricelli A, Pifferi A, Spinelli L, Cubeddu R: Clinical trial of timeresolved scanning optical mammography at 4 wavelengths between 683 and $975 \mathrm{~nm} ., \mathrm{J}$. Biomed. Opt. 2004, 9:464-473

60. Barbour RL, Schmitz CH, Klemer DP, Pei Y, Graber HL: Design and initial testing of system for simultaneous dynamic optical tomographic mammography. Edited by Washington, DC, Optical Society of America, 2004, p. WD4

61. Jiang $\mathrm{H}, \mathrm{Xu} \mathrm{Y,} \mathrm{Iftimia} \mathrm{N,} \mathrm{Eggert} \mathrm{J,} \mathrm{Klove} \mathrm{K,} \mathrm{Baron} \mathrm{L,} \mathrm{Fajardo} \mathrm{L:} \mathrm{Three-dimensional}$ optical tomographic imaging of breast in a human subject IEEE Trans. Med. Imaging 2001, 20:1334-1340

62. Chernomordik V, Hattery D, Pifferi A, Taroni P, Torricelli A, Valentini G, Cubeddu R, Gandjbakhche AH: A random walk methodology for quantification of the optical 
characteristics of abnormalities embedded within tissue-like phantoms, Optics Letters 2000, 25:951

63. Chernomordik V, Hattery DW, Grosenick D, Wabnitz H, Rinneberg H, Moesta KT, Schlag PM, Gandjbakhche A: Quantification of optical properties of a breat tumor using random walk theory, J. Biomed. Optics 2002, 7:80-87

64. Grosenick D, Wabnitz H, Moesta KT, Mucke J, Moller M, Stroszczynski C, Stossel J, Wassermann B, Schlag PM, Rinneberg H: Concentration and oxygen saturation of haemoglobin of 50 breast tumours determined by time-domain optical mammography., Phys. Med. Biol. 2004, 49:1165-1181

65. Grosenick D, Wabnitz H, Rinneberg H, Moesta KT, Schlag PM: Development of a timedomain optical mammograph and first in vivo applications, J. Biomed. Optics 1999, 1:342355

66. Pogue BW, Zhu H, Nwaigwe C, McBride TO, Osterberg UL, Paulsen KD, Dunn JF: Hemoglobin imaging with hybrid magnetic resonance and near-infrared diffuse tomography., Adv. Exp. Med. Biol. 2003, 530:215-224

67. Yates T, Hebden JC, Gibson A, Everdell N, Arridge SR, Douek M: Optical tomography of the breast using a multi-channel time-resolved imager, Phys. Med. Biol. 2005, 50:25032517

68. Schmidt F, Fry M, Hillman E, Hebden J, Delpy D: A 32-channel time-resolved instrument for medical optical tomography, Rev. Sci. Instrum. 2000, 71:256-265

69. Arridge SR, Schweiger M: Image reconstruction in optical tomography, Philosophical Transactions of the Royal Society of London Series B-Biological Sciences 1997, 352:717726

70. Arridge S, Hebden J, Schweiger M, Schmidt F, Fry M, Hillman E, Dehghani H, Delpy D: A method for 3D time-resolved optical tomography, Int. J. Imaging Syst. Technol. 2000, $11: 2-11$

71. Srinivasan S, Pogue BW, Brooksby B, Jiang S, Dehghani H, Kogel C, Wells WA, Poplack SP, Paulsen KD: Near-Infrared Characterization of Breast Tumors In-Vivo using Spectrally-Constrained Reconstruction, TCRT 2005, 4:513-526

72. McBride TO, Pogue BW, Jiang J, Österberg UL, Paulsen KD: A parallel-detection frequency-domain nearinfrared tomography system for hemoglobin imaging of the breast in vivo, Rev. Sci. Instrumen. 2001, 72:1817-1824

73. Conti PS, Lilien DL, Hawley K, Keppler J, Grafton ST, Bading JR: PET and [18F]-FDG in oncology: A clinical update, Nuclear Medicine and Biology 1996, 23:717

74. Zhu Q, Huang M, Chen N, Zarfos K, Jagjivan B, Kane M, Hedge P, Kurtzman S: Ultrasound-guided optical tomographic imaging of malignant and benign breast lesions: initial clinical results of 19 cases, Neoplasia 2003, 5:379-388

75. Gulsen G, Yu H, Wang J, Nalcioglu O, Merritt S, Bevilacqua F, Durkin AJ, Cuccia DJ, Lanning R, Tromberg BJ: Congruent MRI and near-infrared spectroscopy for functional and structural imaging of tumors., Technol. Cancer Res. Treat. 2002, 1:497-505

76. Ntziachristos V, Yodh AG, Schnall MD, Chance B: MRI-Guided diffuse optical spectroscopy of malignant and benign breast lesions, Neoplasia 2002, 4:347

77. Vishwanath K, Pogue B, Mycek MA: Quantitative fluorescence lifetime spectroscopy in turbid media: comparison of theoretical, experimental and computational methods, Physics in Medicine and Biology 2002, 47:3387-3405 
78. Goertzen AL, Meadors AK, Silverman RW, Cherry SR: Simultaneous molecular and anatomical imaging of the mouse in vivo, Phys. Med. Biol. 2002, 47:4315

79. Pogue BW, Paulsen KD: High-resolution near-infrared tomographic imaging simulations of the rat cranium by use of a priori magnetic resonance imaging structural information, Optics Letters 1998, 23:1716

80. Vauhkonen M, Vadasz D, Kaipio JP, Somersalo E, Karjalainen PA: Tikhonov regularization and prior information in electrical impedance tomography, IEEE transactions on medical imaging 1998, 17:285

81. Borsic A, Lionheart WRB, McLeod CN: Generation of Anisotropic-Smoothness Regularization Filters for EIT, IEEE transactions on medical imaging 2002, 21:579

82. Ouyang X, Wong WH, Johnson VE, Hu X, Chen C: Incorporation of correlated structural images in PET image reconstruction, IEEE transactions on medical imaging 1994, 13:627

83. Glidewell $\mathrm{M}, \mathrm{Ng} \mathrm{KT}$ : Anatomically constrained electrical impedance tomography for anisotropic bodies via a twostep approach, IEEE Tras. Med. Imag. 1995, 14:498

84. Dickin F, Wang M: Electrical resistance tomography for process tomography, Meas. Sci. Tech. 1996, 7:247

85. Loke MH: Electrical imaging surveys for environmental and engineering studies. Edited by $1997, \mathrm{p}$

86. Brooksby B, Jiang S, Dehghani H, Pogue BW, Paulsen KD, Weaver J, Kogel C, Poplack SP: Combining near infrared tomography and magnetic resonance imaging to study in vivo breast tissue: implementation of a Laplacian-type regularization to incorporate MR structure, J. Biomed Opt. 2005, 10:51504

87. Hsiang D, Shah N, Yu H, Su M, Cerussi A, Butler J, Baick C, Mehta R, Nalcioglu O, Tromberg B: Coregistration of dynamic contrast enhanced MRI and broadband diffuse optical spectroscopy for characterizing breast cancer., TCRT 2005, 4:549-558

88. Andrews R, Mah R, Da Silva L: The NASA smart probe project for real-time multiplemicrosensor tissue recognition, SPIE Proceedings 2004, 5326:92-97

89. Zhu C, Labawy C, Burnside E, Harter J, Ramanujam N: Development of optical sensor for spectroscopic detection of breast cancer during needle biopsy, Lasers in Surgery and Medicine 2005, 6: 